\title{
Aplicando Ecossistemas de Software na Disciplina Engenharia de Software
}

\author{
Francisco Victor da Silva Pinheiro \\ Universidade Federal do Ceará - Campus Quixadá \\ Quixadá, Ceará, Brasil \\ victor.pinheiro.ce@alu.ufc.br
}

Ecossistemas de Software (ECOS) são um conjunto de soluções de software que permitem automatizar atividades e transações pelos atores em um ecossistema social ou de negócios e as organizações que oferecem essas soluções [2]. O ECOS consiste em um conjunto de atores interagindo como uma unidade, que por sua vez interagem com um mercado distribuído entre software e serviços, juntamente com as relações entre as mais variadas entidades [9], colaborando em conjunto com outras entidades em uma plataforma tecnológica central. Esses elementos formam um ECOS e requerem a integração de mecanismos e ferramentas para apoiar a troca de informações, recursos e artefatos, bem como para assegurar a comunicação e interação dos desenvolvedores e usuários [3].

$\mathrm{Na}$ literatura existe uma lacuna no ensino de ECOS devido à carência por modelos e exemplos [8][6], sendo um interessante complemento ao ensino em Engenharia de Software. Sendo assim, sua aplicação na educação em Computação propicia uma visão mais global dos relacionamentos entre fornecedores e clientes ao redor de uma plataforma central.

Nesse contexto, este trabalho propõe uma pesquisa para a aplicação de ECOS em Engenharia de Software, visando a identificação de plataformas centrais, relacionamentos e modelagem, relacionando com outras áreas sempre que possível.

A proposta em si necessita da definição de uma metodologia de ensino de ECOS, a princípio a ser aplicada na disciplina de Engenharia de Software, mas como o tema possui características interdisciplinares, é possível aplicar em outras disciplinas ou em conjunto, por exemplo a disciplina de Análise e Projeto de Sistemas.

Para o ensino de ECOS na disciplina Engenharia de Software, um conjunto de atividades foi planejado. A atividade inicial consiste do planejamento do ensino de ECOS dentro da disciplina de Engenharia de Software, ocorrendo no início do semestre letivo. As atividades em si sobre ECOS se iniciam conforme o cronograma da disciplina, compostas pela aula expositiva de ECOS, um exercício em sala de aula de modelagem preliminar e o início de um exercício a ser desenvolvido fora da sala de aula, mais completo e com entrega posterior, com feedbacks iniciais para os alunos sobre ECOS. Em seguida, ao longo da disciplina, os alunos desenvolvem atividades de modelagem e refinamento do ECOS, com a aplicação de um questionário online (após o exercício fora da sala de aula) ao final, consolidação das respostas e dos exercícios, análise e interpretação

Fica permitido ao(s) autor(es) ou a terceiros a reprodução ou distribuição, em parte ou no todo, do material extraído dessa obra, de forma verbatim, adaptada ou remixada, bem como a criação ou produção a partir do conteúdo dessa obra, para fins não comerciais, desde que sejam atribuídos os devidos créditos à criação original, sob os termos da licença CC BY-NC 4.0 .

EduComp'21, Abril 26-30, 2021, Jataí, Goiás, Brasil (On-line)

(C) 2021 Copyright mantido pelo(s) autor(es). Direitos de publicação licenciados à Sociedade Brasileira de Computação (SBC).

\author{
Emanuel Ferreira Coutinho \\ Universidade Federal do Ceará - Campus Quixadá \\ Quixadá, Ceará, Brasil \\ emanuel.coutinho@ufc.br
}

dos dados, e por fim, os refinamentos para as próximas edições da disciplina.

Adicionalmente, uma ferramenta para suportar a modelagem de ECOS está em desenvolvimento, sendo que já funcional, baseada na notação SSN (Software Supply Network - Rede de Suprimento de Software) [4], a notação mais popularmente utilizada pela comunidade de ECOS para a modelagem. O SSN é uma série de software, hardware e organizações de serviços ligados, que cooperam para atender às demandas do mercado [5]. Os elementos gráficos da notação auxiliam na representação dos atores e os comportamentos e interações dos mesmos dentro do ecossistema. Assim, com a utilização da ferramenta é possível deixar o ensino de ECOS mais prático e aplicado, possibilitando uma melhor adequação da metodologia.

A pesquisa já foi aplicada a turmas de Engenharia de Software da graduação e da pós-graduação [1][7], com validações iniciais. Aspectos de conceitos e modelagem foram desenvolvidos e analisados. Análises qualitativas por meio de Grounded Theory foram executadas. Uma ferramenta para suportar a modelagem de ECOS já foi aplicada algumas vezes em sala de aula, com a obtenção de feedbacks sobre a abordagem planejada. Adicionalmente, elementos de experiência do usuário foram avaliados.

Os próximos passos da pesquisa são: refinar a metodologia de ensino de ECOS na disciplina de Engenharia de Software, ampliar as funcionalidades da ferramenta, e aplicar em sala de aula. Uma ideia para ampliar o estudo seria empregar metodologias ativas no ensino, como sala de aula invertida e aprendizagem baseada em problemas. Espera-se que assim os benefícios de se estudar ECOS colaborem para um aprendizado em Engenharia de Software com uma maior qualidade.

Por fim, com esta pesquisa tem-se a intenção de fortalecer a área de ECOS, prover uma ferramenta para a comunidade aplicar no ensino e possibilitar a documentação de ECOS, disseminar o conhecimento na área e promover pesquisa colaborativa na comunidade de ECOS e de Engenharia de Software.

\section{REFERÊNCIAS}

[1] Igor R. Alencar, Emanuel F. Coutinho, Leonardo O. Moreira, and Carla I. M. Bezerra. 2020. A Tool for Software Ecosystem Models: An Analysis on theirImplications in Education. In Proceedings of the XXXIV Brazilian Symposium on Software Engineering (Natal, Brazil) (SBES 2020). ACM, New York, NY, USA, 10 pages. https://doi.org/10.1145/3422392.3422486

[2] Jan Bosch. 2009. From Software Product Lines to Software Ecosystems. In Proceedings of the 13th International Software Product Line Conference (San Francisco, California, USA) (SPLC '09). Carnegie Mellon University, USA, 111-119.

[3] Jan Bosch. 2011. Software ecosystems-implications for strategy, business model and architecture. In 2011 15th International Software Product Line Conference. IEEE, 351-351.

[4] Vasilis Boucharas, Slinger Jansen, and Sjaak Brinkkemper. 2009. Formalizing Software Ecosystem Modeling. In Proceedings of the 1st International Workshop on Open Component Ecosystems (Amsterdam, The Netherlands) (IWOCE '09). Association for Computing Machinery, New York, NY, USA, 41-50. https: //doi.org/10.1145/1595800.1595807 
[5] Gabriella Costa, Felyppe Silva, Rodrigo Santos, Cláudia Werner, and Toacy Oliveira 2013. From Applications to a Software Ecosystem Platform: An Exploratory Study In Proceedings of the Fifth International Conference on Management of Emergent Digital EcoSystems (Luxembourg, Luxembourg) (MEDES '13). ACM, New York, NY, USA, 9-16. https://doi.org/10.1145/2536146.2536159

[6] Emanuel Coutinho, Italo Santos, Leonardo Moreira, and Carla Bezerra. 2018 Um Estudo Preliminar de Ecossistemas de Software na Disciplina Engenharia de Software. Anais do Workshop de Informática na Escola 24, 1 (2018), 21-30. https://doi.org/10.5753/cbie.wie.2018.21

[7] Emanuel F. Coutinho, Italo Santos, Leonardo O. Moreira, and Carla I. M. Bezerra. 2019. A Report on the Teaching of Software Ecosystems in Software Engineering Discipline. In Proceedings of the XXXIII Brazilian Symposium on Software
Engineering (Salvador, Brazil) (SBES 2019). ACM, New York, NY, USA, 130-139. https://doi.org/10.1145/3350768.3351302

[8] Emanuel Ferreira Coutinho, Davi Viana, and Rodrigo Pereira dos Santos. 2017. An Exploratory Study on the Need for Modeling Software Ecosystems: The Case of SOLAR SECO. In 9th International Workshop on Modelling in Software Engineering (MISE) (Buenos Aires, Argentina) (MISE '17). IEEE Press, Piscataway, NJ, USA, 47-53. https://doi.org/10.1109/MiSE.2017.3

[9] Slinger Jansen, Sjaak Brinkkemper, and Anthony Finkelstein. 2009. Business Network Management as a Survival Strategy: A Tale of Two Software Ecosystems. Iwseco@Icsr 2009 (2009). 